\title{
Predictors of hospitalization for lower respiratory tract infection in children aged $<2$ years in the province of Quebec, Canada
}

\author{
Z. ZHOU ${ }^{1}$, R. GILCA ${ }^{2}$, G. DECEUNINCK ${ }^{1}$, F. D. BOUCHER ${ }^{3}$, H. CHAREST ${ }^{4}$ \\ AND P. DE WALS ${ }^{5} *$ \\ ${ }^{1}$ Quebec University Hospital Research Center, Quebec City, Canada \\ ${ }^{2}$ Direction des Risques biologiques et Santé au Travail, Institut national de Santé publique du Québec, Quebec \\ City, Canada \\ ${ }^{3}$ Departement of Pediatrics, Laval University, Quebec City, Canada \\ ${ }^{4}$ Laboratoire de Santé publique du Québec, Institut national de Santé publique du Québec, Sainte-Anne-de- \\ Bellevue, Canada \\ ${ }^{5}$ Departement of Social and Preventive Medicine, Laval University, Quebec City, Canada
}

Received 11 July 2015; Final revision 19 August 2015; Accepted 1 September 2015;

first published online 18 September 2015

\section{SUMMARY}

Young age, adverse environmental conditions and infectious agents are established risk factors of lower respiratory tract infection (LRTI), whereas pneumococcal conjugate vaccines may be protective. To explore their relative role as predictors of hospitalizations under the continental climate prevailing in the province of Quebec, Canada, an ecological study was performed. Records with a main diagnosis of LRTI in children born during 2007-2010 and observed up to their second-year anniversary were extracted from the provincial hospital administrative database. Respiratory virus surveillance data and statistics on ambient air temperature were obtained. Vaccine use in different birth cohorts was derived from the Quebec City Immunization Registry. Additive and multiplicative Poisson regression models were applied to estimate attributable fractions. Age, month of birth, ambient temperature, and respiratory syncytial virus (RSV), human metapneumovirus (hMPV) and influenza-positive test proportions were significant predictors of LRTI hospitalizations. No substantial differences were observed in cohorts exposed to the 7-valent or 10-valent pneumococcal conjugate vaccines. In the additive model, the fraction of hospitalizations explained by temperature variation was 37\%, whereas RSV circulation explained $28 \%$, hMPV $4 \%$ and influenza $1 \%$. Complex interplay between biological, environmental and social mechanisms may explain the important role of ambient air temperature in predicting LRTI hospitalization risk in young children.

Key words: Ambient temperature, ecological study, hospital admissions, respiratory infections, respiratory virus, pneumococcal conjugate vaccine.

\section{INTRODUCTION}

Lower respiratory tract infection (LRTI) is a major cause of hospitalization in children worldwide [1, 2]. A marked seasonal variation is observed in countries situated outside the equatorial belt [3-5]. Age is a well-established risk factor of the risk and severity of infections [6-8].

\footnotetext{
* Author for correspondence: Professor P. De Wals, Département de médecine sociale et préventive, Université Laval, IUCPQ, 2725 Chemin Sainte-Foy, Québec (QC) G1V4G5, Canada. (Email: philippe.dewals@criucpq.ulaval.ca)
} 
Respiratory pathogens including influenza A and B viruses, respiratory syncytial virus (RSV), human metapneumovirus (hMPV) and Streptococcus pneumoniae cause diseases with different epidemiological patterns and clinical presentations [9]. In the last decade, influenza and pneumococcal conjugate vaccines have been introduced in immunization programmes for children in order to reduce the burden of LRTI [10-12]. In the province of Quebec, Canada, the 7-valent (PCV-7), 10-valent (PCV-10) and 13-valent (PCV-13) pneumococcal conjugate vaccines have been sequentially used in the publicly funded immunization programme [13, 14]. The relative contribution of all these factors in causing severe LRTI leading to hospitalization is not known. The objective of this ecological study was to assess the relative contribution of age, daily ambient temperature, circulation of respiratory viruses and pneumococcal conjugate vaccines as predictors of hospitalization for LRTI in children aged $<2$ years, born during 2007-2010, in Quebec, a province experiencing a continental climate mostly.

\section{METHODS}

\section{Study population}

The study population included children residing in the province of Quebec, born between 1 January 2007, and 31 December 2010, and observed up to their second-year anniversary.

\section{Data sources}

The monthly number of births was obtained from the Quebec Statistics Institute. A uniform distribution of births by day of month was assumed and personmonths of observations were computed assuming a steady-state population from birth up to age 24 months.

In Quebec, all residents are insured for health services and all acute care hospitals are public. All hospital discharges are registered in the administrative electronic database MedEcho [15]. A main diagnosis and up to 15 secondary diagnoses are extracted from medical charts by certified archivists and coded according to the 10th Revision of the International Classification of Diseases (ICD-10). A list of records pertaining to hospital admissions with a main code of LRTI between 1 January 2007 and 29 February 2012 was obtained (details in Supplementary Table S1). Children born between 1 January 2007 and 28 February 2010 had a 24-month follow-up whereas observations were truncated for those born later. Transfers and multiple admissions within a 7-day period were considered as a single episode.

In Quebec, pneumococcal conjugate vaccines are recommended at age 2, 4, 6 and 12 months for low-risk children, whereas four doses are recommended for children at high risk of pneumococcal disease [16]. The distribution of pneumococcal vaccine doses was obtained from the Quebec City Immunization Registry [17]. The main vaccines used for the primary immunization series $(\geqslant 90 \%$ of first, second and third doses administered up to age 11 months) and toddler booster dose ( $\geqslant 90 \%$ third and fourth doses administered at age 12 months or after) in each monthly birth cohort were identified (details in Supplementary Table S2).

The Quebec Public Health Institute Laboratory Surveillance Network routinely reports statistics on the number and percentage of positive respiratory virus assays. Approximately half of hospital microbiology laboratories $(n=45 / 94)$, including all four tertiary paediatric centres in the Province of Quebec participate in the respiratory virus surveillance programme. Diagnostic tests include rapid antigen detection tests (RADT), nucleic acid tests (NAT) and viral cultures. The number of tests and the number of positive results for each respiratory virus are compiled on a weekly basis and submitted to the Provincial Reference Laboratory [18]. In the present study, weekly percentages of positive tests for influenza (A and B), RSV and hMPV during the years 2007-2012 were used as a proxy of the intensity of virus circulation in the population.

Daily ambient air temperature average statistics in the central town of Trois-Rivières were provided by the Meteorological Service of Environment Canada [19]. A 7-day moving average was used as a proxy for climate exposure.

\section{Statistical analyses}

Statistical analyses were performed using SAS version 9.3 software (SAS Institute, USA) with a significance threshold set at 0.05 (two-sided tests). Potential predictors of daily hospital admissions rates were first tested in univariate analyses. Univariate associations were measured by Pearson and Spearman correlation coefficients $(\rho)$ and linear regression and determination coefficients $\left(\beta\right.$ and $R^{2}$ ).

Multivariate analyses were restricted to children aged 3-23 months as respiratory infections in the early neonatal period are often related to prenatal or perinatal causes, are difficult to diagnose and no effect of pneumococcal vaccination could be expected. First 


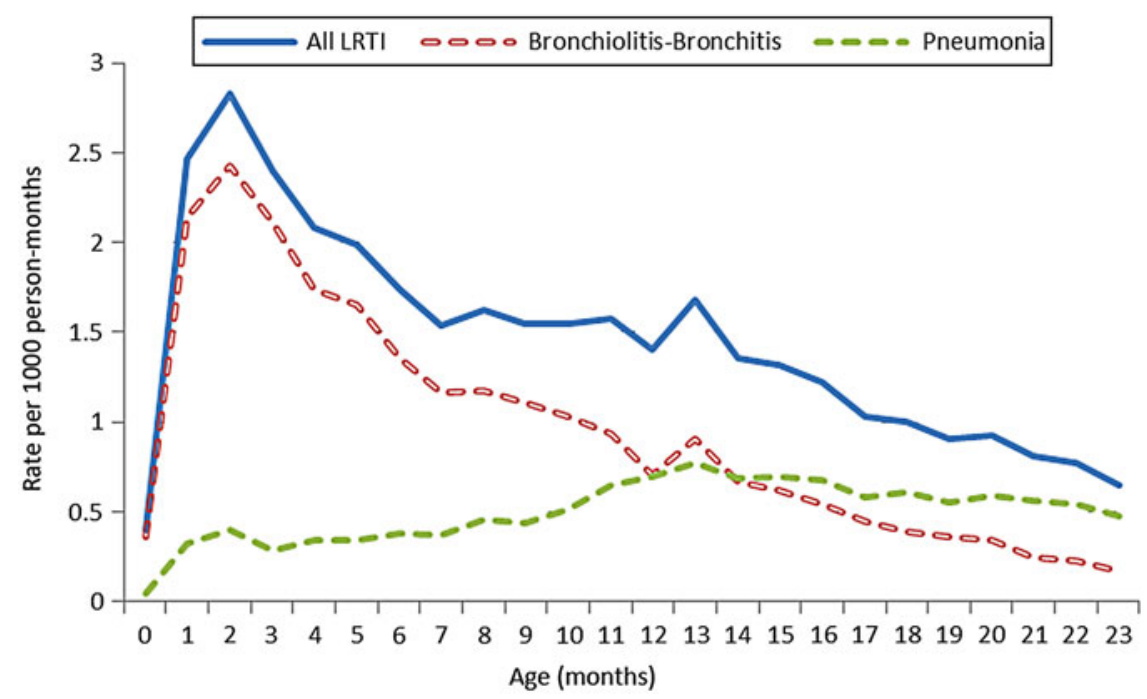

Fig. 1. Frequency rate of hospital admissions for lower respiratory tract infection (LRTI) according to age, in children born between 1 January 2007 and 28 February 2010, in the province of Quebec, Canada (those with 24-month follow-up).

hospitalizations were modelled using both identity-link additive and log-link multiplicative Poisson regression models [20]. All hospitalizations were modelled using multiplicative models only as convergence could not be reached in additive models. Dependence between repeated hospitalizations for the same patients was taken into account using Generalized Estimating Equations (GEE) [21].

The estimated number of hospital cases attributable to a particular predictor was calculated as the difference between the overall number of hospitalizations in the study population predicted in the model and the estimated number when the variable of interest was set at its lowest possible value. The fraction attributable to each predictor was then calculated by dividing the number of cases attributed to the predictor by the number of total cases.

\section{Ethical approval}

The study protocol was approved by the Quebec University Hospital Research Ethics Board, and access to nominative information in provincial databases was authorized by the Commission d'accès à l'information du Québec, a governmental organization regulating access to documents held by public bodies and the protection of personal information.

\section{RESULTS}

A total of 11361 LRTI episodes in 9742 children were recorded in an estimated population of 349645 children representing a total of 8024120 personmonths of observation. Out of these, $8470(86.9 \%)$ children had a single episode of hospitalization, 1216 $(12.5 \%)$ had two or three episodes and $56(0.6 \%)$ had four or more. One child with 10 episodes had underlying congenital heart disease. Bronchiolitis and bronchitis represented the most frequent diagnostic category ( $n=7461,65.6 \%$ of total), followed by pneumonia ( $n=3896,34 \cdot 3 \%$ of total). In the bronchiolitis/bronchitis category, bronchiolitis was the most frequent condition $(n=7070,94 \cdot 8 \%)$. Of pneumonia cases, unspecified pneumonia was the most frequent diagnostic category $(n=3004,77 \cdot 1 \%$ of total $)$. There were only four cases of empyema.

Hospital admission rates according to age and diagnostic category are shown in Figure 1 (analysis restricted to children born between 1 January 2007 and 18 February 2010, with a 24-month follow-up). The LRTI rate increased from birth to reach a peak of $2 \cdot 8 / 1000$ person-months at age 2 months, and progressively decreased thereafter. This pattern was attributable mostly to the bronchiolitis group. The peak of pneumonia was at age 13 months. The overall cumulative LRTI frequency rate was $3 \cdot 5 \%$ from birth up to the second-year anniversary.

LRTI hospitalization rates showed a marked seasonal pattern with a peak in February and a minimum in July (Fig. 2).

Viral surveillance data are displayed in Table 1 and Figure 3. RSV was detected during long periods with winter peaks during which $20-40 \%$ of tests were positive. Influenza viruses had also a clear seasonal 


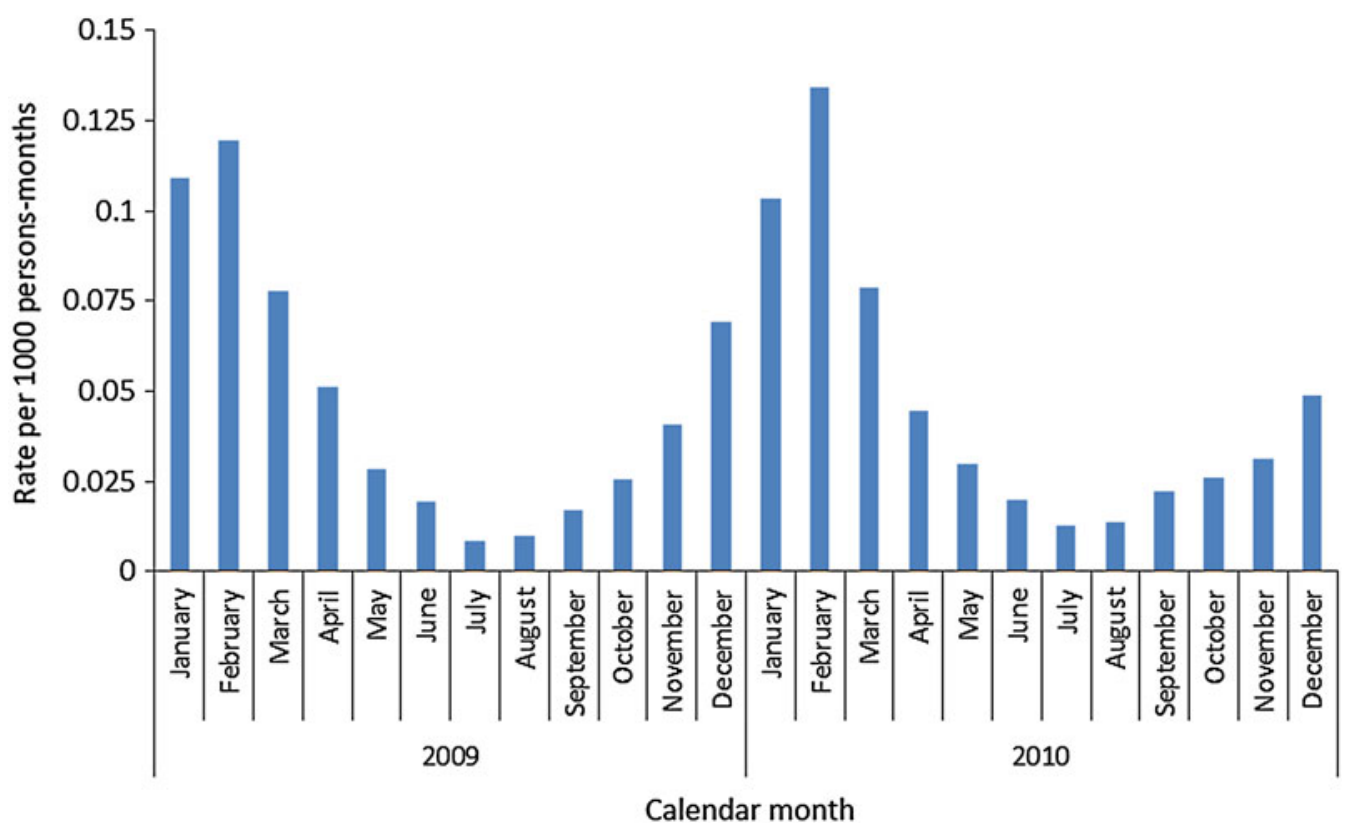

Fig. 2. Frequency rate of hospital admissions for lower respiratory tract infection according to calendar month, in children born between 1 January 2007 and 28 February 2010, in the province of Quebec, Canada (those with 24-month follow-up).

pattern with much shorter periods of circulation and a lower proportion of positive tests. The 2009 influenza pandemic year was atypical with two peaks, the first during spring and the second during autumn, whereas almost no influenza circulation was detected in 2010 . The daily average ambient temperature recorded in Trois-Rivières ranged between a maximum of $28.4^{\circ} \mathrm{C}$ and a minimum of $-26.7^{\circ} \mathrm{C}$ (Table 1). Weekly averages were less extreme with a marked seasonal pattern (Fig. 3).

In univariate analyses of LRTI hospitalization rates, statistically significant associations were seen for ambient temperature, RSV, influenza, but not for hMPV. Details are shown in Supplementary Figure S1.

In multivariate analyses (Table 2), viral circulation, temperature, age, and month of birth were significant predictors of LRTI admission. There was no significant difference between different PCV schedules with one exception: PCV-13 use was associated with a lower risk compared to PCV-7 use in the additive model and in the multiplicative model for first hospitalizations. The effect of age was for pneumonia and bronchiolitis. PCV-13 use was not associated with a reduced risk of bronchiolitis. The remaining results were similar for pneumonia and bronchiolitis (details in Supplementary Tables S3 and S4).

The proportions of hospitalizations explained by variation in temperature and viral circulation are given in Table 3. Temperature was the most important predictor of hospitalizations for LRTI, explaining between $37 \%$ and $50 \%$ of episodes. Of viruses, RSV was by far the most important contributor, explaining between $28 \%$ and $34 \%$ of admissions. The role of influenza was modest, explaining only $1-6 \%$ of admissions. A similar ranking was observed for the predictors of pneumonia and bronchiolitis, and details are presented in Supplementary Tables S5 and S6.

\section{DISCUSSION}

Ecological studies have been widely used in epidemiology to explore aetiological hypotheses but results should be interpreted with caution [22]. In our study, hospitalization risk was measured at the individual level whereas potential predictors (covariates) were measured at the ecological level. In order to explore the role of different predictors, both additive and multiplicative regression models were used, giving consistent results in terms of ranking of coefficients. It has been suggested that additive models provide more valid attributable fraction estimates than multiplicative models [23, 24]. Indeed, multiplicative models generating curvilinear dose-response relationships tended to overestimate the contribution of continuous variables as opposed to categorical variables. 
Table 1. Weekly positive test percentages for respiratory viruses in Quebec, and average ambient air temperature in Trois-Rivières, between 1 January 2007 and 29 February 2012

\begin{tabular}{lllll}
\hline \hline Variable & Mean (s.D.) & Median & Minimum & Maximum \\
\hline Respiratory syncytial virus & $9 \cdot 4(9 \cdot 6)$ & $5 \cdot 2$ & 0 & $38 \cdot 9$ \\
Influenza A \& B & $5 \cdot 1(6 \cdot 8)$ & $1 \cdot 1$ & 0 & $26 \cdot 1$ \\
Human metapneumovirus & $2 \cdot 4(3 \cdot 1)$ & $1 \cdot 1$ & 0 & $19 \cdot 1$ \\
Daily average temperature $\left({ }^{\circ} \mathrm{C}\right)$ & $6 \cdot 0(11 \cdot 7)$ & $6 \cdot 6$ & $-26 \cdot 7$ & $28 \cdot 4$ \\
7-day moving temperature $\left({ }^{\circ} \mathrm{C}\right)$ & $6 \cdot 0(11 \cdot 3)$ & $6 \cdot 6$ & $-18 \cdot 2$ & $25 \cdot 2$ \\
\hline \hline
\end{tabular}
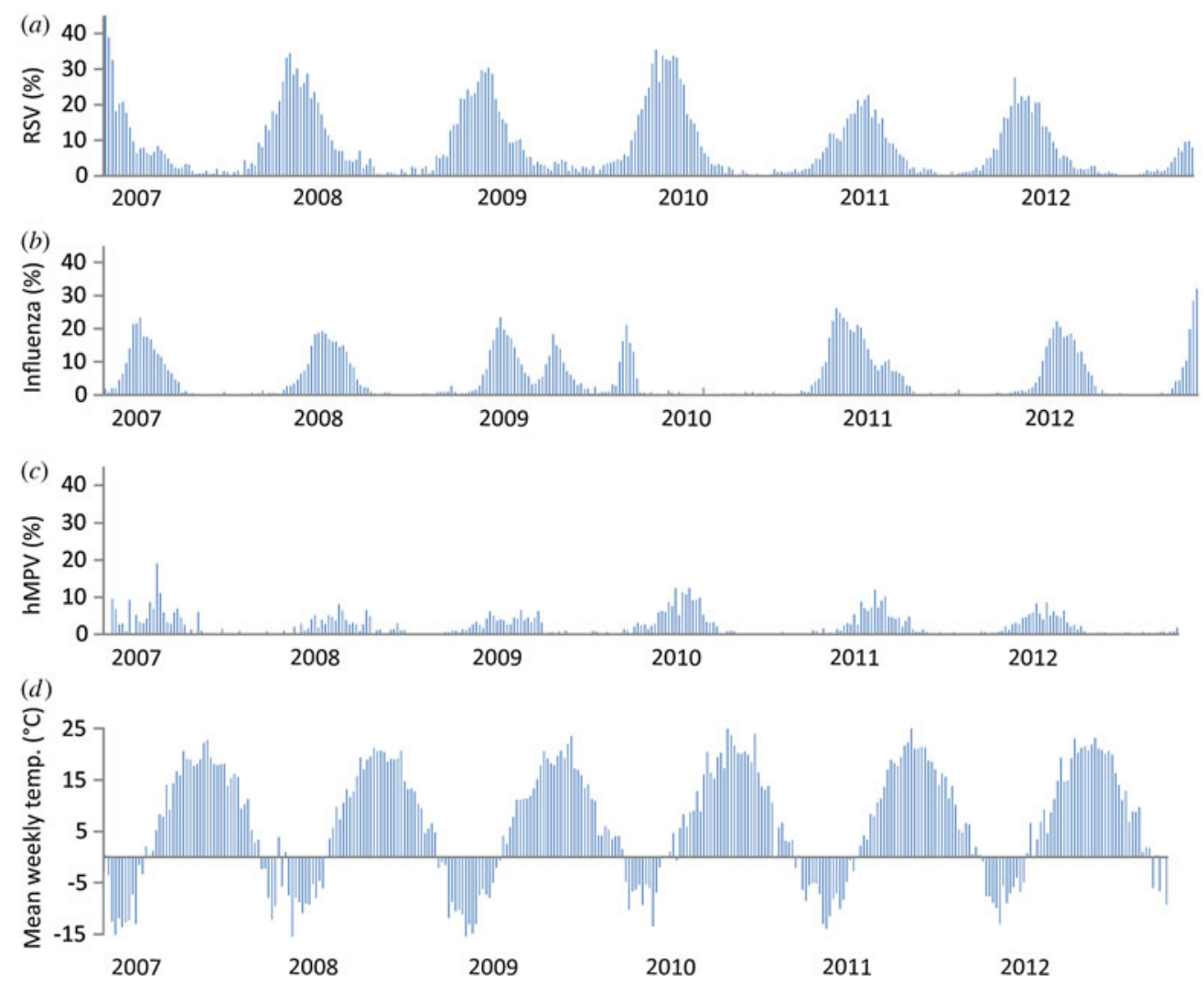

Fig. 3. Intensity of circulation of respiratory viruses (weekly percentage of positive tests recorded by the Quebec National Public Health Laboratory), including (a) respiratory syncytial virus (RSV), (b) influenza A \& B, (c) human metapneumovirus (hMPV), and (d) ambient air temperature (weekly average ambient air temperature recorded in Trois-Rivières), in Quebec, 2007-2013.

The main analysis was performed for LRTI although similar predictors and ranking were found for diagnostic subcategories of pneumonia and bronchiolitis. The age distribution of admissions for bronchiolitis with a peak at age 2 months is congruent with other observations and may be explained, in part, by RSV infections occurring at early age [6, 25-27]. The peak of pneumonia hospitalizations at age 13 months is more difficult to explain and some degree of misclassification cannot be excluded in younger children. Many infectious agents are involved in the aetiology of pneumonia and results of microbiological investigations cannot accurately predict clinical and radiological findings [28]. Committee guidelines and expert opinions for diagnostic investigations in young children admitted for respiratory infection usually recommend standard chest X-ray examination, but not all of them [29, 30]. The interpretation of chest X-ray in infants is especially difficult and standard criteria as proposed by the WHO are not used in 
Table 2. Predictors of hospitalizations for lower respiratory tract infection in children born between 1 January 2007 and 31 December 2010, aged $>90$ days and $<2$ years, in the province of Quebec, Canada

\begin{tabular}{|c|c|c|c|c|c|c|}
\hline \multirow[b]{3}{*}{ Predictors } & \multirow{2}{*}{\multicolumn{2}{|c|}{$\frac{\text { Additive Poisson model* }}{\text { First hospitalizations }}$}} & \multicolumn{4}{|c|}{ Multiplicative Poisson model $\dagger$} \\
\hline & & & \multicolumn{2}{|c|}{$\begin{array}{l}\text { First } \\
\text { hospitalizations }\end{array}$} & \multicolumn{2}{|c|}{ All hospitalizations } \\
\hline & Coefficient & $P$ & $\mathrm{RR}$ & $P$ & $\mathrm{RR}$ & $P$ \\
\hline \multicolumn{7}{|l|}{ Viral circulation } \\
\hline Respiratory syncytial virus & $0 \cdot 007$ & $<0 \cdot 0001$ & $1 \cdot 03$ & $<0.0001$ & $1 \cdot 03$ & $<0 \cdot 0001$ \\
\hline Human metapneumovirus & $0 \cdot 004$ & $<0 \cdot 0001$ & $1 \cdot 04$ & $<0 \cdot 0001$ & $1 \cdot 05$ & $<0 \cdot 0001$ \\
\hline Influenza & $0 \cdot 001$ & $0 \cdot 11$ & $1 \cdot 01$ & $<0.0001$ & $1 \cdot 01$ & $<0 \cdot 0001$ \\
\hline Temperature $\left({ }^{\circ} \mathrm{C}\right) \S$ & $0 \cdot 005$ & $<0 \cdot 0001$ & $1 \cdot 03$ & $<0.0001$ & $1 \cdot 03$ & $<0 \cdot 0001$ \\
\hline \multicolumn{7}{|l|}{ Age (months) } \\
\hline $3-5$ & $0 \cdot 131$ & $<0 \cdot 0001$ & $2 \cdot 56$ & $<0 \cdot 0001$ & $2 \cdot 18$ & $<0 \cdot 0001$ \\
\hline $6-8$ & $0 \cdot 076$ & $<0 \cdot 0001$ & 1.94 & $<0 \cdot 0001$ & $1 \cdot 76$ & $<0 \cdot 0001$ \\
\hline $9-11$ & $0 \cdot 068$ & $<0 \cdot 0001$ & $1 \cdot 82$ & $<0.0001$ & $1 \cdot 70$ & $<0 \cdot 0001$ \\
\hline $12-17$ & $0 \cdot 050$ & $<0 \cdot 0001$ & $1 \cdot 49$ & $<0 \cdot 0001$ & $1 \cdot 41$ & $<0 \cdot 0001$ \\
\hline $18-23$ & Ref. & - & Ref. & - & Ref. & - \\
\hline \multicolumn{7}{|l|}{ Month of birth } \\
\hline January-September & Ref. & - & Ref. & - & Ref. & - \\
\hline October-December & $0 \cdot 022$ & $<0 \cdot 0001$ & $1 \cdot 19$ & $<0 \cdot 0001$ & $1 \cdot 29$ & $<0 \cdot 0001$ \\
\hline Hospitalization year (2007-2012) & $-0 \cdot 010$ & $0 \cdot 11$ & $0 \cdot 94$ & $0 \cdot 06$ & $0 \cdot 95$ & $0 \cdot 35$ \\
\hline \multicolumn{7}{|l|}{ Main vaccines $\|$} \\
\hline $\mathrm{PCV}-7+\mathrm{PCV}-7$ & Ref. & - & Ref. & - & Ref. & - \\
\hline $\mathrm{PCV}-7+\mathrm{PCV}-7 / \mathrm{PCV}-10$ & $-0 \cdot 006$ & $0 \cdot 57$ & $0 \cdot 96$ & $0 \cdot 45$ & $0 \cdot 91$ & $0 \cdot 50$ \\
\hline PCV-7 + PCV-10 & $0 \cdot 019$ & $0 \cdot 06$ & $1 \cdot 03$ & $0 \cdot 56$ & $0 \cdot 98$ & $0 \cdot 84$ \\
\hline PCV-7/PCV-10 + PCV-10 & $0 \cdot 024$ & $0 \cdot 06$ & $1 \cdot 06$ & $0 \cdot 38$ & $0 \cdot 97$ & $0 \cdot 80$ \\
\hline PCV-10 + PCV-10 & $0 \cdot 043$ & $0 \cdot 003$ & $1 \cdot 08$ & $0 \cdot 26$ & $0 \cdot 99$ & $0 \cdot 93$ \\
\hline PCV-10 + PCV-10/PCV-13 & $0 \cdot 020$ & $0 \cdot 22$ & $1 \cdot 15$ & $0 \cdot 09$ & $1 \cdot 12$ & $0 \cdot 48$ \\
\hline PCV-10 + PCV-13 & $0 \cdot 013$ & $0 \cdot 50$ & $1 \cdot 08$ & $0 \cdot 41$ & $0 \cdot 98$ & $0 \cdot 88$ \\
\hline PCV-10/PCV-13 + PCV-13 & $0 \cdot 014$ & $0 \cdot 53$ & $1 \cdot 01$ & $0 \cdot 92$ & 0.94 & $0 \cdot 74$ \\
\hline PCV-13 + PCV-13 & $-0 \cdot 080$ & $<0.0001$ & $0 \cdot 76$ & $0 \cdot 003$ & $0 \cdot 73$ & $0 \cdot 11$ \\
\hline
\end{tabular}

RR, Rate ratio.

* Additive Poisson model assumes a linear relationship between intensity of exposure and response.

$\dagger$ Multiplicative Poisson model implies an exponential relationship between intensity of exposure and response.

$\$$ Weekly percentage of positive tests.

§7-day moving average ambient air temperature (inversed) recorded in Trois-Rivières.

$\| \geqslant 90 \%$ of doses administered in each monthly birth cohort for the primary infant series + toddler booster.

routine practice [31]. Clinicians may be prone to select bronchiolitis as a main diagnosis for any LRTI in children aged $<1$ year. For this reason, it would be wise to assess the global impact of pneumococcal vaccines in Phase IV studies using the LRTI category instead of the usual all-cause pneumonia category [32].

A major finding in our study was the predominant role of ambient air temperature and the previous 7-day moving average in predicting hospital admissions for LRTI. In the province of Quebec, $90 \%$ of the population resides in the Montreal-Trois-Rivières-Quebec City-St Laurence corridor. Ambient air temperature values recorded in Trois-Rivières (study period average $6.0^{\circ} \mathrm{C}$ ) were slightly higher than in Quebec City $\left(4.7^{\circ} \mathrm{C}\right)$ and lower than in Montreal $\left(6.9^{\circ} \mathrm{C}\right)$, but correlations in daily averages in the three cities were very high (coefficient $0.99, P<0 \cdot 0001$ ). Absolute ambient air temperature or its variation may be directly linked to the incidence and severity of respiratory infections through irritation of the respiratory tract mucosa $[3,33]$. In addition, temperature may be a proxy for other environmental factors including light intensity, humidity, and air pollution [34, 35]. Temperature may also be associated with other seasonal influences such as the quality of innate and acquired immunity, or circulation of unmeasured or unknown respiratory pathogens. It is important to note that temperature also affects human behaviours and living conditions 
Table 3. Fraction of hospitalizations for lower tract respiratory infections predicted by viral circulation and ambient temperature in additive and multiplicative models

\begin{tabular}{|c|c|c|c|c|c|c|}
\hline \multirow[b]{3}{*}{ Predictors } & \multirow{2}{*}{\multicolumn{2}{|c|}{$\frac{\text { Additive model* }}{\text { First hospitalizations }}$}} & \multicolumn{4}{|c|}{ Multiplicative model $\dagger$} \\
\hline & & & \multicolumn{2}{|c|}{ First hospitalizations } & \multicolumn{2}{|c|}{ All hospitalizations } \\
\hline & $\%$ & $(95 \% \mathrm{CI})$ & $\%$ & $(95 \% \mathrm{CI})$ & $\%$ & $(95 \% \mathrm{CI})$ \\
\hline \multicolumn{7}{|l|}{ Viral circulation } \\
\hline Respiratory syncytial virus & $27 \cdot 5$ & (23.6 to $31 \cdot 4)$ & $35 \cdot 4$ & $(32 \cdot 1-38 \cdot 6)$ & $34 \cdot 3$ & $(30 \cdot 4-37 \cdot 9)$ \\
\hline Human metapneumovirus & $3 \cdot 8$ & $(2 \cdot 1$ to $5 \cdot 5)$ & $11 \cdot 1$ & $(9 \cdot 1-12 \cdot 8)$ & $12 \cdot 4$ & $(10 \cdot 3-14 \cdot 3)$ \\
\hline Influenza & $1 \cdot 4$ & $(-0 \cdot 4$ to $3 \cdot 1)$ & $6 \cdot 1$ & $(3 \cdot 9-8 \cdot 1)$ & $5 \cdot 4$ & $(2 \cdot 9-7 \cdot 8)$ \\
\hline Temperature $\left({ }^{\circ} \mathrm{C}\right) \S$ & $37 \cdot 1$ & $(30 \cdot 1$ to $43 \cdot 3)$ & $47 \cdot 2$ & $(42 \cdot 2-51 \cdot 9)$ & $49 \cdot 6$ & $(44 \cdot 4-54 \cdot 2)$ \\
\hline
\end{tabular}

CI, Confidence interval.

* Additive Poisson model assumes a linear relationship between intensity of exposure and response.

$\dagger$ Multiplicative Poisson model implies an exponential relationship between intensity of exposure and response.

$\$$ Weekly percentage of positive tests.

$\S 7$-day moving average ambient air temperature (inversed) recorded in Trois-Rivières.

such as time spent outdoors or indoor crowding [36]. Finally, temperature represents a general background variation that was previously modelled using periodic functions [37], but real temperature data are more appropriate, especially for respiratory infections. More studies are needed to better understand the role of temperature in the occurrence of respiratory infections. Results of our study may also have implications for the short-term planning of hospital services in Quebec which usually relies on alerts generated by respiratory virus surveillance, but not so much on climatic conditions.

In Quebec, respiratory virus surveillance is based on tests routinely prescribed in a large sample of hospitals, including all four tertiary pediatric centres [18]. The weekly percentage of positive tests was considered as a more valid indicator of viral circulation than the absolute number of positive tests, which is more sensitive to variation in practice. Three viruses only were included in this study because the surveillance of other respiratory pathogens (i.e. adenoviruses and parainfluenza viruses) was less reliable during the study years. RSV surveillance mostly resulted from tests in young infants whereas influenza surveillance encompasses all age groups. This may be a problem as the peak of influenza generally occurs earlier in school-aged children than in younger children or elderly adults $[38,39]$. In the additive model, RSV circulation accounted for $28 \%$ of first hospitalizations, while influenza activity accounted for $1 \%$ only. RSV is considered as the main cause of hospital admissions for respiratory infections in young children [40, 41].
In an ecological study in the United States based on viral surveillance data, it was found that RSV was associated with 16 times more hospitalizations than influenza in children aged $<1$ year, and the ratio was $5: 1$ in children aged $1-4$ years [42].

In a systematic review of randomized trials, cohort and case-control studies of inactivated influenza vaccines in children, the authors concluded that vaccine effectiveness in preventing influenza cases was demonstrated for children aged $\geqslant 2$ years, but not for those aged $<2$ years [43]. In Quebec, influenza vaccination has been recommended for all children aged 6-23 months since 2004, but not for older children [44]. Results of immunization surveys in Quebec showed low coverage rates, peaking at $35 \%$ in 2006 , and falling to $16 \%$ in 2011 [45]. A marked impact of influenza vaccination in reducing the burden of LRTI admissions in young children is thus unlikely. With a very low frequency of hospital admissions in the 6-24 months age group potentially explained by influenza infections, the relevance of the current recommendation is questionable. Clearly, more effective influenza vaccines are needed and a RSV vaccine should be a research priority.

In our study, surveillance data on invasive pneumococcal disease (IPD) in young children was not selected as a potential predictor of LRTI hospitalizations. As reported in a previous study [46], the IPD epidemiology in Quebec has been markedly influenced by the introduction of pneumococcal conjugate vaccines. IPD incidence in children aged $<5$ years was $67 / 100000$ in $2001-2004$, and decreased to $32 / 100$ 
000 in 2007-2009 following the introduction of PCV-7 in December 2004. A further decrease to $24 / 100000$ was observed in 2010-2011 following the introduction of PCV-10 in June 2009. Variations of this magnitude are not expected for LTRIs, all-cause pneumonia or non-bacteraemic pneumococcal pneumonia, a highly unreliable diagnostic category in hospital administrative databases [28]. Moreover, seasonal variation in IPD in children is less marked than for respiratory viruses [37].

The effectiveness of PCV-7 and PCV-10 in preventing all-cause pneumonia has been demonstrated in randomized trials, but not for PCV-13 however [47-49]. Following the implementation of routine infant vaccination with PCV-7 in Quebec in December $2004(2+1$ doses for low-risk children and $3+1$ doses for high-risk infants), a 13\% decrease in hospital admissions for allcause pneumonia was observed [14]. In the present analysis, the reference group is the cohort of children exposed to PCV-7. Since the implementation of the PCV programme, approximately $90 \%$ of children are now receiving the recommended number of doses by age 2 years, and the coverage has been stable over years [45]. As shown in the present study, the introduction of PCV-10 in 2009, was not followed by a further decrease in hospital admissions for LRTI and all-cause pneumonia. A lower frequency of first admissions was seen in cohorts exposed to PCV-13. This trend should be confirmed as the number of children in the PCV-13-only cohort was limited $(n=14175)$, and the truncated observation period was much shorter than for the other vaccine schedules (median 14.9 months vs. 24 months). Our plan is to extend the analysis to include cohorts of children not exposed to any pneumococcal conjugate vaccines and additional cohorts exposed to PCV-13.

\section{CONCLUSION}

Results of ecological studies should be interpreted with care and our study is no exception. The predominant role of ambient temperature in predicting the risk of hospitalization for LRTI in young children is an interesting finding and causal mechanisms should be explored further. Replicating the analysis in a region experiencing other climatic conditions would be interesting. Of respiratory viruses, RSV seems to be, by far, the most important pathogen, whereas the role of influenza viruses appears to be minor in this age group.

\section{SUPPLEMENTARY MATERIAL}

For supplementary material accompanying this paper visit http://dx.doi.org/10.1017/S0950268815002204.

\section{ACKNOWLEDGEMENTS}

The authors thank Professor Michel Carbon, Department of Mathematics \& Statistics, Laval University, for statistical advice.

The study was supported by an investigatorinitiated research grant from GlaxoSmithKline Biologicals. The sponsor had no role in the design and conduct of the study, collection, management, analysis, and interpretation of the data, and preparation, review, and approval of the manuscript.

\section{DECLARATION OF INTEREST}

Philippe De Wals received research grants and reimbursement of travel expenses from GlaxoSmithKline, Pfizer and Novartis. Rodica Gilca received research grants from Pfizer and Sanofi-Pasteur. The remaining authors do not have a commercial or other association that might pose a conflict of interest.

\section{REFERENCES}

1. Rudan I, et al. Epidemiology and etiology of childhood pneumonia. Bulletin of the World Health Organization 2008; 86: 408-416.

2. Nair H, et al. Global and regional burden of hospital admissions for severe acute lower respiratory infections in young children in 2010: a systematic analysis. Lancet 2013; 381: 1380-1390.

3. Makinen TM, et al. Cold temperature and low humidity are associated with increased occurrence of respiratory tract infections. Respiratory Medicine 2009; 103: 456462.

4. Tang JW, Loh TP. Correlations between climate factors and incidence $-\mathrm{a}$ contributor to RSV seasonality. Reviews in Medical Virology 2014; 24: 15-34.

5. te Beest DE, et al. Driving factors of influenza transmission in the Netherlands. American Journal of Epidemiology 2013; 178: 1469-1477.

6. Tulloh RM, Bury S. Prevention and prophylaxis of respiratory syncytial virus in pediatric cardiology: a UK perspective. Future Cardiology 2014; 10: 235-242.

7. Geevarghese B, Weinberg A. Cell-mediated immune responses to respiratory syncytial virus infection: Magnitude, kinetics, and correlates with morbidity and age. Human Vaccines \& Immunotherapeutics 2014; 10: $1047-1056$.

8. Takeyama A, et al. Clinical and epidemiologic factors related to subsequent wheezing after virus-induced lower respiratory tract infections in hospitalized 
pediatric patients younger than 3 years. European Journal of Pediatrics 2014; 173: 959-966.

9. Chang AB, et al. Improving the diagnosis, management, and outcomes of children with pneumonia: where are the gaps? Frontiers in Pediatrics 2013; 1: 29.

10. Kellner J. Update on the success of the pneumococcal conjugate vaccine. Paediatrics \& Child Health 2011; 16: $233-240$.

11. Ladhani SN, et al. Invasive pneumococcal disease after routine pneumococcal conjugate vaccination in children, England and Wales. Emerging Infectious Diseases 2013; 19: 61-68.

12. Hoen AG, et al. Effect of expanded US recommendations for seasonal influenza vaccination: comparison of two pediatric emergency departments in the United States and Canada. Canadian Medical Association Journal 2011; 183: E1025-1032.

13. De Wals $\mathbf{P}$, et al. Invasive pneumococcal diseases in birth cohorts vaccinated with PCV-7 and/or PHiD-CV in the province of Quebec, Canada. Vaccine 2012; 30: 6416-6420.

14. De Wals P, et al. Pneumonia after implementation of the pneumococcal conjugate vaccine program in the province of Quebec, Canada. Pediatric Infectious Disease Journal 2008; 27: 963-968.

15. Ministère de la Santé et des Services sociaux. Normative framework of the MedÉcho system. MSSS, October 1987, revision April 2014 (http://publications.msss. gouv.qc.ca/acrobat/f/documentation/2000/00-601.pdf). Accessed 1 August 2015.

16. Quebec Immunization Committee. Assessment of the appropriateness of an immunization program for pneumococcal infections in children using a reduced number of doses of conjugate vaccine. Institut national de santé publique, 2005 (https://www.inspq.qc.ca/pdf/publications/ 391-ImmunizationConjugateVaccine.pdf). Accessed 1 August 2015.

17. De Wals P, et al. Uptake of pneumococcal conjugate vaccine: methodological issues in measurement and impact of publicly funded programs. Canadian Journal of Public Health 2009; 100: 413-416.

18. Institut National de Santé Publique du Québec. Influenza surveillance. INSPQ, 2013 (http://www.inspq.qc.ca/ influenza/surveillance-de-1-influenza). Accessed 1 August 2015.

19. Environment Canada. National Climate Data and Information Archive. 2013. (http://climate.weather.gc. ca/index_e.html). Accessed 9 September 2013.

20. Coughlin SS, et al. Regression methods for estimating attributable risk in population-based case-control studies: a comparison of additive and multiplicative models. American Journal of Epidemiology 1991; 133: 305-313.

21. Guo Z, Gill TM, Allore HG. Modeling repeated time-to-event health conditions with discontinuous risk intervals. An example of a longitudinal study of functional disability among older persons. Methods of Information in Medicine 2008; 47: 107-116.

22. Rothman KJ, Greenland S, Lash TL. Ecologic studies. Modern Epidemiology. Philadelphia: Lippincott Williams \& Wilkins, 2008, pp. 596-619.
23. Boshuizen HC, Feskens EJ. Fitting additive Poisson models. Epidemiologic Perspectives \& Innovations 2010; 7: 4.

24. Gay NJ, et al. Estimating deaths due to influenza and respiratory syncytial virus. Journal of the American Medical Association 2003; 289: 2499; author reply 500-502.

25. Leader S, Kohlhase K. Respiratory syncytial virus-coded pediatric hospitalizations, 1997 to 1999. Pediatric Infectious Disease Journal 2002; 21: 629-632.

26. Shay DK, et al. Bronchiolitis-associated hospitalizations among US children, 1980-1996. Journal of the American Medical Association 1999; 282: 1440-1446.

27. Ogra PL. Respiratory syncytial virus: the virus, the disease and the immune response. Paediatric Respiratory Reviews 2004; 5 (Suppl. A): S119-S126.

28. Moore HC, et al. Use of data linkage to investigate the aetiology of acute lower respiratory infection hospitalisations in children. Journal of Paediatrics and Child Health 2012; 48: 520-528.

29. Ralston SL, et al. Clinical practice guideline: the diagnosis, management, and prevention of bronchiolitis. Pediatrics 2014; 134: e1474-1502.

30. Swingler GH, Hussey GD, Zwarenstein M. Randomised controlled trial of clinical outcome after chest radiograph in ambulatory acute lower-respiratory infection in children. Lancet 1998; 351: 404-408.

31. Pneumonia Vaccine Trial Investigators' Group. Standardization of interpretation of chest radiographs for the diagnosis of pneumonia in children. Department of Vaccines and Biologicals, World Health Organization, 2001.

32. Grijalva CG, Griffin MR. Population-based impact of routine infant immunization with pneumococcal conjugate vaccine in the USA. Expert Review of Vaccines 2008; 7: 83-95.

33. Falagas ME, et al. Effect of meteorological variables on the incidence of respiratory tract infections. Respiratory Medicine 2008; 102: 733-737.

34. Kim PE, et al. Association of invasive pneumococcal disease with season, atmospheric conditions, air pollution, and the isolation of respiratory viruses. Clinical Infectious Diseases 1996; 22: 100-106.

35. Nicoli EJ, et al. Influenza and RSV make a modest contribution to invasive pneumococcal disease incidence in the UK. Journal of Infection 2013; 66: 512-520.

36. Ampofo $\mathbf{K}$, et al. Seasonal invasive pneumococcal disease in children: role of preceding respiratory viral infection. Pediatrics 2008; 122: 229-237.

37. Weinberger DM, et al. Seasonal drivers of pneumococcal disease incidence: impact of bacterial carriage and viral activity. Clinical Infectious Diseases 2014; 58: 188-194.

38. Peters TR, et al. Relative timing of influenza disease by age group. Vaccine 2014; 32: 6451-6456.

39. Sebastian R, et al. Age-related trends in the timeliness and prediction of medical visits, hospitalizations and deaths due to pneumonia and influenza, British Columbia, Canada, 1998-2004. Vaccine 2008; 26: 1397-1403. 
40. Santibanez $\mathbf{P}$, et al. Acute care utilization due to hospitalizations for pediatric lower respiratory tract infections in British Columbia, Canada. BMC Health Services Research 2012; 12: 451.

41. Murray J, et al. Risk factors for hospital admission with RSV bronchiolitis in England: a population-based birth cohort study. PLoS ONE 2014; 9: e89186.

42. Zhou H, et al. Hospitalizations associated with influenza and respiratory syncytial virus in the United States, 19932008. Clinical Infectious Diseases 2012; 54: 1427-1436.

43. Jefferson T, et al. Vaccines for preventing influenza in healthy children. Cochrane Database of Systematic Reviews 2012; 8: CD004879.

44. Gilca R, et al. Statement on the relevance of including children aged 24-59 months in the Quebec immunization program against influenza. Québec: Institut national de santé publique, 2013 (http://www.inspq.qc.ca/ pdf/publications/1654_AvisPertAjoutEnfants24-59Mois PQVI.pdf). Accessed 1 August 2015.

45. Boulianne N, et al. Survey on vaccination coverage of children aged 1-2 years in 2012, in Quebec. Québec: Institut national de santé publique du Québec, 2013. (http://www. inspq.qc.ca/pdf/publications/1651_EnquCouvVaccinEnfa nts1Et2Ans2012.pdf). Accessed 1 August 2015.

46. De Wals $\mathbf{P}$, et al. Impact of $2+1$ pneumococcal conjugate vaccine program in the province of Quebec, Canada. Vaccine 2014; 32: 1501-1506.

47. Tregnaghi MW, et al. Efficacy of pneumococcal nontypable Haemophilus influenzae protein $\mathrm{D}$ conjugate vaccine (PHiD-CV) in young Latin American children: a double-blind randomized controlled trial. PLoS Medicine 2014; 11: e1001657.

48. Palmu AA, et al. Effect of pneumococcal Haemophilus influenzae protein $\mathrm{D}$ conjugate vaccine (PHiD-CV10) on outpatient antimicrobial purchases: a double-blind, cluster randomised phase 3-4 trial. Lancet Infectious Diseases 2014; 14: 205-212.

49. Black SB, et al. Effectiveness of heptavalent pneumococcal conjugate vaccine in children younger than five years of age for prevention of pneumonia. Pediatric Infectious Disease Journal 2002; 21: 810815. 